\title{
Spaces of complex functions and vector measures in incomplete spaces ${ }^{1}$
}

\author{
Werner Ricker and Martin Väth \\ (Communicated by Jürgen Appell)
}

2000 Mathematics Subject Classification. 28B05, 39B12, 46E30.

Keywords and phrases. Vector measures, space of integrable functions.

\begin{abstract}
It is known that the space $L^{1}(\mu)$ of complex functions which are integrable with respect to a vector measure $\mu$ taking values in a (not necessarily complete) locally convex space is not an ideal, in general. We discuss several natural properties which $L^{1}(\mu)$ may or may not possess and consider various implications between these properties. For a particular class of properties, whether or not there exists a particular space of the form $L^{1}(\mu)$ having these properties, is shown to be equivalent to the existence of any space of complex functions on $\mathbb{C}$ having these same properties.
\end{abstract}

\section{Introduction}

Let $(S, \Sigma)$ be a measurable space with a fixed ideal of null-sets. By $\mathfrak{M}$, we denote the corresponding space of $\mathbb{C}$-valued $\Sigma$-measurable functions (with the usual identification of a.e. identical functions). Let $X \subseteq \mathfrak{M}$ be

\footnotetext{
${ }^{1}$ The paper was completed in the framework of a Heisenberg Fellowship (Az. VA 206/1-1) of the second author. Financial support by the DFG is gratefully acknowledged.
} 
a (complex) linear subspace. We are interested in connections between the following properties of $X$ :

$$
\begin{gathered}
f \in X \Longrightarrow|f| \in X, \\
f \in X \Longrightarrow \bar{f} \in X, \\
f \in \mathfrak{M} \text { and }|f| \in X \Longrightarrow f \in X, \\
f \in \mathfrak{M} \text { and }|f| \leq g \in X \Longrightarrow f \in X, \\
L^{\infty}(\Sigma) \subseteq X .
\end{gathered}
$$

We consider (1) and (2) as special cases of the property

$$
f \in X \Longrightarrow h \circ f \in X
$$

for some (appropriate) function $h$, and many of our results will apply to this more general property. Of course, one is usually interested in $h$ being a Borel function, say, and not too degenerate, so that condition (6) has a reasonable chance of being satisfied and non-trivial.

By the (complex) linearity of $X$, we may replace $\bar{f}$ in (2) by either $\operatorname{Re} f$ or $\operatorname{Im} f$ to obtain an equivalent condition. Clearly, all the above properties (1)(5) hold if $X$ contains the constant functions and if it is solid, i.e. if the implication

$$
(|f| \leq|g| \quad \text { and } \quad g \in X) \Longrightarrow f \in X
$$

holds. However, the properties are not independent from each other. For example, it is clear that $X$ is solid if and only if (1) and (4) hold simultaneously. Moreover, (4) clearly implies (3) and (if $X$ contains the constant functions) also (5). No other implications of the above properties are obvious.

The space $X=L^{1}(\mu)$ of integrable functions with respect to a vector measure $\mu$ on $(S, \Sigma)$ is of particular interest and is the main motivation of this note. For the definition and basic properties of such spaces we refer to [1] and the references therein, for example. The space $L^{1}(\mu)$ always contains constant functions and, in fact, all $\Sigma$-simple functions. If the space in which $\mu$ assumes its values is sequentially complete and the fixed ideal of null-sets consists of the $\mu$-null sets, then it is well known that $L^{1}(\mu)$ is solid, and so all of the above properties are satisfied. However, if we omit the completeness assumption, then $L^{1}(\mu)$ need not be solid. Moreover, for each of the above properties (1)-(5) it is known that there exists a vector measure $\mu$, defined on the Borel subsets of $[0,1]$, such that $L^{1}(\mu)$ fails to have the corresponding property; see [1]. The corresponding examples given in [1] show additionally that, except for the above mentioned (obvious) implications, none of the above properties implies any of the others with three exceptions. These examples do not disprove that (3) implies (5) or even that (3) implies (4), and they do not disprove that (1) implies (2). The purpose of this paper is to 
show that the first two of these implications are always true (in any space $X$ of complex functions). Moreover, we provide a somewhat surprising result which shows that implications like $(1) \Longrightarrow(2)$, both involving properties of type (6) for appropriate $h$, fail for some particular space of the kind $X=L^{1}(\mu)$ if (and only if) there already exists any linear space of complex functions for which they fail.

Perhaps some explanation is needed of why property (4) is of particular interest for the space $X=L^{1}(\mu)$.

Proposition 1 (cf. [1]). . The space $X=L^{1}(\mu)$ satisfies (4) if and only if it has the Lebesgue convergence property, that is, if and only if for any sequence of $\mu$-integrable functions $\left(f_{n}\right)_{n}$ which satisfies $\left|f_{n}\right| \leq g$ for some integrable $g \geq 0$ and converges a.e. to some $f$, the limit function $f$ belongs to $L^{1}(\mu)$ and $f_{n} \rightarrow f$ with respect to the topology of $L^{1}(\mu)$.

\section{Conditions (3) and (4) are equivalent}

As already noted, always $(4) \Longrightarrow(3)$. We now show that $(3) \Longrightarrow(4)$ holds in every (complex) linear space $X \subseteq \mathfrak{M}$. As a special case we also obtain, of course, that $(3) \Longrightarrow(5)$ (provided that $X$ contains the constant functions); for the special case when $X=L^{1}(\mu)$, the latter was already observed in [1].

Lemma 1. Let $f: S \rightarrow \mathbb{C}$ and $g: S \rightarrow[0, \infty)$ be measurable functions satisfying $|f(x)| \leq g(x)$. Then there exist measurable functions $\rho_{1}, \rho_{2}: S \rightarrow$ $\mathbb{R}$ such that $f(x)=g(x)\left(e^{i \rho_{1}(x)}+e^{i \rho_{2}(x)}\right)$.

Proof. Since $f(x)=|f(x)| e^{i \rho(x)}$ for some measurable function $\rho$, it is no loss of generality to assume that $f=|f| \geq 0$. Then $f=g \cdot \varphi$ for some measurable function $\varphi: S \rightarrow[0,1]$. Then $\rho_{1}(x):=-\rho_{2}(x):=$ $\arccos (\varphi(x) / 2)$ have the required properties.

Proposition 2. For any complex linear space $X \subseteq \mathfrak{M}$ the properties (3) and (4) are equivalent.

Proof. Assume that (3) holds. Given $f, g \in \mathfrak{M}$ satisfying $|f| \leq g \in X$, let $\rho_{1}, \rho_{2}$ be as in Lemma 1. By assumption, the functions $g \cdot e^{i \rho_{k}}(k=1,2)$ belong to $X$ and hence, so does their sum $f$.

We obtain immediately the following consequences which may be surprising at a first glance.

Corollary 1. A complex linear space $X \subseteq \mathfrak{M}$ is solid if and only if for any $f \in \mathfrak{M}$ the equivalence

$$
f \in X \Longleftrightarrow|f| \in X
$$


holds.

Corollary 2. Let $\mu$ be a vector measure. The (complex) space $X=L^{1}(\mu)$ possesses property (3) if and only if it possesses the Lebesgue convergence property.

\section{Equivalences to implications concerning properties of the form (6)}

Let $h_{1}, h_{2}: \mathbb{C} \rightarrow \mathbb{C}$ be two functions. We are interested in the existence of a counterexample to the implication

$$
\text { (6) holds for } h=h_{1} \Longrightarrow \text { (6) holds for } h=h_{2} \text {. }
$$

In other words, we look for a set $M$ and a complex linear space $X$ of complex functions $f: M \rightarrow \mathbb{C} \quad$ (later also for $X=L^{1}(\mu)$ for a vector measure $\mu$ ) which simultaneously satisfies the following two conditions:

$$
f \in X \Longrightarrow h_{1} \circ f \in X \text {. }
$$

Of particular interest: does there exist a space of the form $X=L^{1}(\mu)$ which possesses the above two properties?

The following notation will be convenient. Given a function $f: M \rightarrow \mathbb{C}$, we denote by $[f]$ the smallest complex linear space of functions in $\mathbb{C}^{M}$ which contains $f$ and satisfies the condition

$$
g \in[f] \Longrightarrow h_{1} \circ g \in[f] .
$$

Necessarily, $X:=[f]$ satisfies (8). Moreover, the space $[f]$ always exists; it can be described in a constructive way as follows.

Proposition 3. Given a function $f: M \rightarrow \mathbb{C}$, define inductively $[f]_{1}:=$ $\{f\}$ and $[f]_{n+1}:=\operatorname{span}\left([f]_{n} \cup\left\{h_{1} \circ g: g \in[f]_{n}\right\}\right)$, and put $[f]_{\infty}:=\bigcup_{n=1}^{\infty}[f]_{n}$. Then $[f]=[f]_{\infty}$.

Proof. By induction, each complex linear subspace of $\mathbb{C}^{M}$ containing $f$ and satisfying (10) must contain each $[f]_{n}$. It thus suffices to prove that $[f]_{\infty}$ is a linear space with the property that $g \in[f]_{\infty}$ implies $h_{1} \circ g \in[f]_{\infty}$. Evidently, both are true by construction.

By $I$, we denote the identity function on $\mathbb{C}$. Consider the condition

$$
h_{2} \notin[I] \subseteq \mathbb{C}^{\mathbb{C}} .
$$

Note that the space $[I]$ depends only on $h_{1}$ so that (11) can be interpreted as some sort of "algebraic independence" of the functions $h_{1}$ and $h_{2}$. Probably, the most convenient way to verify (11) for concrete functions $h_{1}$ and $h_{2}$ is 
to find a property which all functions from $[I]$ have in common but, which $h_{2}$ fails to possess.

Example 1. Let $h_{1}(z)=\bar{z}$. Induction on $n$ shows that all functions in $[I]_{n}$ (and thus all functions in $[I]_{\infty}=[I]$ ) are real-differentiable, additive, positively homogeneous, and odd. Although the function $h_{2}(z)=|z|$ is positively homogeneous, it is neither additive nor odd nor real-differentiable at 0 . These are three reasons why $h_{2}$ does not belong to $[I]$, i.e. that (11) holds.

Condition (11) is a natural one for our considerations as the following result shows.

Theorem 1. For functions $h_{1}, h_{2}: \mathbb{C} \rightarrow \mathbb{C}$ the following three conditions are equivalent.

1. There exists a set $M$ and a complex linear function space $X \subseteq \mathbb{C}^{M}$ satisfying (8) and (9).

2. Condition (11) holds.

3. There exists $H \in[I]$ such that $h_{2} \circ H \notin[I]$.

The key observation to the proof of Theorem 1 is the following fact.

Lemma 2. Let $M \neq \emptyset$ and $G: M \rightarrow \mathbb{C}$ be a function. Then $[G]_{n} \subseteq \mathbb{C}^{M}$ consists of precisely those functions of the form $f \circ G$ with $f \in[I]_{n} \subseteq \mathbb{C}^{\mathbb{C}}$. In particular, the functions in $[G]$ are precisely those functions of the form $f \circ G$ with $f \in[I]$.

Proof. We prove the claim by induction on $n$. The case $n=1$ is obvious, since $G=I \circ G$. Assume that the claim has already been proved for $n$.

Let $g \in[G]_{n+1}$ be given. By definition of $[G]_{n+1}$, we can find finitely many $g_{j} \in[G]_{n}$ and complex numbers $\lambda_{j} \in \mathbb{C}$ such that

$$
g=\lambda_{1} g_{1}+\cdots+\lambda_{k} g_{k}+\lambda_{k+1}\left(h_{1} \circ g_{k+1}\right)+\cdots+\lambda_{m}\left(h_{1} \circ g_{m}\right) .
$$

By the inductive assumption, there exist $f_{j} \in[I]_{n}$ with $g_{j}=f_{j} \circ G$. It follows that

$$
f:=\lambda_{1} f_{1}+\cdots+\lambda_{k} f_{k}+\lambda_{k+1}\left(h_{1} \circ f_{k+1}\right)+\cdots+\lambda_{m}\left(h_{1} \circ f_{m}\right)
$$

satisfies $g=f \circ G$. Moreover, $f \in[I]_{n+1}$ by construction.

Conversely, given some $f \in[I]_{n+1}$, we can find finitely many $f_{j} \in[I]_{n}$ and complex numbers $\lambda_{j} \in \mathbb{C}$ such that (12) holds. By the inductive assumption, each of the functions $f_{j} \circ G$ belongs to $[G]_{n}$, and so the composition

$f \circ G=\lambda_{1}\left(f_{1} \circ G\right)+\cdots+\lambda_{k}\left(f_{k} \circ G\right)+\lambda_{k+1}\left(h_{1} \circ\left(f_{k+1} \circ G\right)\right)+\cdots+\lambda_{m}\left(h_{1} \circ\left(f_{m} \circ G\right)\right)$ belongs to $[G]_{n+1}$.

Proof. [Proof of Theorem 1] Clearly, 2. implies 3. (with $H=I$ ), and 3. implies 1. (with $M=\mathbb{C}$ and $X=[I]$ ). So, assuming that there is a complex 
linear space $X \subseteq \mathbb{C}^{M}$ satisfying (8) and (9), it remains to prove that (11) holds. But, if (11) would fail, i.e. $h_{2} \in[I]$, then Lemma 2 would imply that $h_{2} \circ H \in[H] \subseteq X$, which is a contradiction.

We now address the question of whether the space $X$ in Theorem 1 can be chosen to be of the form $X=L^{1}(\mu)$ for some vector measure $\mu$ defined on a measurable space $(S, \Sigma)$. Some care is needed in this case, because $X$ does not consist of individual functions but rather of equivalence classes of functions. There are several possible definitions for null-functions with respect to vector measures. Fortunately, all these definitions turn out to be equivalent (even in incomplete spaces). To formulate this precisely, recall that a set $E \in \Sigma$ is a $\mu$-null set for a vector measure $\mu$, if $\mu(F)=0$ for each $F \subseteq E$ with $F \in \Sigma$.

Proposition 4. Let $(S, \Sigma)$ be a measurable space and $\mu$ be a vector measure defined on $\Sigma$ and taking values in a locally convex space $Y$ with continuous dual space $Y^{*}$. Then, for each $\Sigma$-measurable function $f: S \rightarrow \mathbb{C}$, the following statements are equivalent:

1. $f^{-1}(\mathbb{C} \backslash\{0\})$ is a $\mu$-null set.

2. $f \in L^{1}(\mu)$ and $\int_{E} f d \mu=0$ for each $E \in \Sigma$.

3. $\int_{S}|f| d|\langle\mu, \ell\rangle|=0$ for each $\ell \in Y^{*}$.

Proof. If 1 . holds, then for each $\ell \in Y^{*}$ the function $f$ is integrable with respect to the complex measure $\langle\mu, \ell\rangle$ and, for each $F \in \Sigma$, we have $\int_{F} f d\langle\mu, \ell\rangle=0$ (because $\mu$-null sets are $\langle\mu, \ell\rangle$-null sets). Hence 2. holds. If 2. is true then, for each $E \in \Sigma$ and each $\ell \in Y^{*}$, we have that $\int_{E} f d\langle\mu, \ell\rangle=0$. This implies (see e.g. [2, Theorem 6.13]) that $\int_{F}|f| d|\langle\mu, \ell\rangle|=0$ for each $F \in \Sigma$, and so 3 . holds. Finally, assume that 1 . fails. Since the $\mu$-null sets form a $\sigma$-ideal, $E_{n}:=|f|^{-1}\left(\left(\frac{1}{n}, \infty\right)\right)$ cannot be $\mu$-null for every $n \in \mathbb{N}$. Hence, there exist some $n$ and some measurable set $F \subseteq E_{n}$ with $\mu(F) \neq 0$, i.e. there is some $\ell \in Y^{*}$ with $c:=\int_{F} d\langle\mu, \ell\rangle \neq 0$. Then $\int_{S}|f| d|\langle\mu, \ell\rangle| \geq n^{-1}|c|>0$, and so 3 . fails.

In view of this result, two measurable functions $f, g: S \rightarrow \mathbb{C}$ are called $\mu$-equivalent, if $\{s: f(s) \neq g(s)\}$ is a $\mu$-null set. For this definition it is clear that our previous results also hold for the corresponding equivalence classes of functions (elements of the space $[f]$ also consist of the corresponding equivalence classes). From now on we will tacitly use this fact without further mentioning it.

In order to find a space $X=L^{1}(\mu)$ as in Theorem 1, it is necessary to require a slightly more restrictive condition than (11). Namely:

For every finite family of closed sets $A_{1}, \ldots, A_{K} \subseteq \mathbb{C}$ with union $\mathbb{C}$ there is some $k$ such that $\left.h_{2}\right|_{A_{k}}$ is not the restriction of any function from $[I] \subseteq \mathbb{C}^{\mathbb{C}}$. 
Unfortunately, it is not so obvious how to verify condition (13). Indeed, for the functions $h_{1}(z)=\bar{z}$ and $h_{2}(z)=|z|$, none of the reasons given for substantiating (11) in Example 1 also establish (13) without further reasoning. For a class of functions (including the above), we will discuss (13) in the next section.

Theorem 2. Let $h_{1}, h_{2}: \mathbb{C} \rightarrow \mathbb{C}$ be continuous and positively homogeneous (of order 1 ), that is, $h_{j}(t z)=t h_{j}(z)$ for all $t \geq 0$ and $z \in \mathbb{C}$. Assume, in addition, that $h_{1}$ satisfies a Lipschitz condition. Then the following statements are equivalent.

1. There exists a measurable space $(S, \Sigma)$ and a vector measure $\mu$ defined on $\Sigma$ such that the space $X:=L^{1}(\mu)$ satisfies (8) and (9).

2. There is a vector measure $\mu$ defined on the Borel sets of $[0,1]$ and taking values in a subspace $Y \subseteq L^{1}([0,1])$ such that the space $X:=L^{1}(\mu)$ satisfies $(5),(8)$, and $(9)$.

3. Condition (13) holds.

The next result shows that the necessity of (13) even holds for arbitrary functions $h_{1}, h_{2}: \mathbb{C} \rightarrow \mathbb{C}$.

Proposition 5. Let $h_{1}, h_{2}: \mathbb{C} \rightarrow \mathbb{C}$ be arbitrary functions. Let $(S, \Sigma)$ be a measurable space. Assume that there is a complex linear space $X \subseteq \mathfrak{M}$ of complex measurable functions satisfying (8) and (9). Assume, in addition, that $X$ has the property that $s \cdot f \in X$ for all $f \in X$ and all $\Sigma$-simple functions s. Then (13) holds even for every finite family of Borel sets $A_{1}, \ldots, A_{K}(K \in \mathbb{N})$.

Proof. Assume that (13) fails for some finite family of Borel sets $A_{1}, \ldots, A_{K}$ with union $\mathbb{C}$, i.e. for each $k=1, \ldots, K$, there is $f_{k} \in[I]$ such that $h_{2}=f_{k}$ on $A_{k}$. Lemma 2 (or Theorem 1) implies that $f_{k} \circ H \in X$ for all $H \in X$. For fixed $H \in X$, we have $h_{2} \circ H=f_{k} \circ H$ on every set $E_{k}:=H^{-1}\left(A_{k}\right) \in \Sigma$. Putting $D_{k}:=E_{k} \backslash \bigcup_{j<k} E_{j} \in \Sigma$, we have $\bigcup_{k=1}^{K} D_{k}=\bigcup_{k=1}^{K} E_{k}=S$, and so $h_{2} \circ H=\sum \chi_{D_{k}} \cdot\left(f_{k} \circ H\right) \in X$. Hence, $X$ does not satisfy (9).

We point out that every space of the kind $X=L^{1}(\mu)$, for any vector measure $\mu$, has the property that $s \cdot f \in X$ whenever $f \in X$ and $s$ is a $\Sigma$-simple function. So, Proposition 5 shows that both implications $1 . \Longrightarrow 3$. and 2. $\Longrightarrow 3$. of Theorem 2 hold. Clearly $2 . \Longrightarrow 1$. also holds.

The rest of this section is concerned with establishing the remaining implication 3. $\Longrightarrow 2$. needed to complete the proof of Theorem 2 .

Proposition 6. If $h_{1}$ is positively homogeneous (resp. continuous), then each space $[I]_{n}$, and thus also $[I]_{\infty}=[I]$, consists only of positively homogeneous (resp. continuous) functions. 
Proof. A straightforward induction on $n$.

Let $\lambda$ denote Lebesgue measure on $[0,1]$, and $\mathfrak{B}$ denote the vector subspace of $L^{1}(\lambda)$ consisting of all Borel simple functions on $[0,1]$, i.e. the space of all complex Borel functions which attain only finitely many values ( $\lambda$-a.e.). In order to construct a vector measure $\mu$ as in Theorem 2, we make use of the following result which is a special case of [1, Corollary 3.2] (with $Y=\mathbb{C}$ and $\mu=\lambda$ ).

Lemma 3. Let $X \subseteq L^{1}(\lambda)$ be a complex linear subspace (equipped with the norm of $L^{1}(\lambda)$ ) which contains the constant functions and has the property that for each $s \in \mathfrak{B}$ and each $f \in X$ also $s \cdot f$ belongs to $X$. Then there is an $X$-valued vector measure $\mu$ defined on the Borel subsets of $[0,1]$ such that $X=L^{1}(\mu)$.

The vector measure in Lemma 3 is simply defined by putting $\mu(E):=$ $\chi_{E} \in X$. Note that $X$ is simultaneously the space in which $\mu$ assumes its values, so that in general both spaces may fail to be sequentially complete.

In the sequel, we consider complex sequences as functions from $\mathbb{N}$ into $\mathbb{C}$. In particular, given a sequence $a=\left(a_{n}\right)_{n}$, the composition $h_{1} \circ a$ is then given by $\left(h_{1}\left(a_{n}\right)\right)_{n}$. Using our previous notation, it thus makes sense to speak of $[a]=[a]_{\infty}$ and $[a]_{n}$.

Lemma 4. Let $h_{1}, h_{2}: \mathbb{C} \rightarrow \mathbb{C}$ be continuous and $a=\left(a_{n}\right)_{n}$ be a sequence in $\mathbb{C}$. Let $\left\{b_{\beta}=\left(b_{\beta, n}\right)_{n}: \beta \in B\right\}$ be a family of sequences in $[a]$ and $\left\{f_{\beta}: \beta \in B\right\}$ be a corresponding family in $[I]$ satisfying $b_{\beta}=f_{\beta} \circ$ a (see Lemma 2) for every $\beta \in B$.

Assume, for each strictly increasing sequence $n_{1}<n_{2}<\cdots$ in $\mathbb{N}$, that there exists a subsequence $\left(n_{j_{m}}\right)_{m}$ and some $\beta \in B$ such that the differences $b_{\beta, n_{j_{m}}}-h_{2}\left(a_{n_{j_{m}}}\right) \rightarrow 0$ as $m \rightarrow \infty$.

Then the set consisting of all limit points of all subsequences of $\left(a_{n}\right)_{n}$ can be written as the union of closed sets $\left\{A_{\beta}: \beta \in B\right\}$ having the property that $\left.h_{2}\right|_{A_{\beta}}=\left.f_{\beta}\right|_{A_{\beta}}$ for each $\beta \in B$.

Proof. By continuity, the sets $A_{\beta}:=\left\{z \in \mathbb{C}: f_{\beta}(z)=h_{2}(z)\right\}$, for $\beta \in B$, are closed. We show that their union contains each limit point $z$ of any subsequence $\left(a_{n_{k}}\right)_{k}$. Indeed, by assumption, we can find a subsequence $\left(n_{j_{m}}\right)_{m}$ and some $\beta \in B$ with $b_{\beta, n_{j_{m}}}-h_{2}\left(a_{n_{j_{m}}}\right) \rightarrow 0$. By continuity of $h_{2}$, we have $h_{2}\left(a_{n_{j_{m}}}\right) \rightarrow h_{2}(z)$ and so, $b_{\beta, n_{j_{m}}} \rightarrow h_{2}(z)$. On the other hand, $b_{\beta, n_{j_{m}}}=f_{\beta}\left(a_{n_{j_{m}}}\right) \rightarrow f_{\beta}(z)$ by continuity of $f_{\beta}$ and so, $f_{\beta}(z)=h_{2}(z)$, i.e. $z \in A_{\beta}$.

For the construction of the space $X$ in part 2. of Theorem 2, we fix a sequence $a=\left(a_{n}\right)_{n}$ in the unit circle $\mathbb{T}:=\{z \in \mathbb{C}:|z|=1\}$ having the property that each point of $\mathbb{T}$ is the limit of some subsequence of $\left(a_{n}\right)_{n}$. 
We also fix a sequence of pairwise disjoint Borel sets $J_{n} \subseteq[0,1]$ of positive Lebesgue measure such that the function

$$
H(x):=\sum_{n=1}^{\infty} n a_{n} \chi_{J_{n}}(x) \quad(x \in[0,1])
$$

belongs to $L^{1}(\lambda)$ (e.g. choose $J_{n}$ such that $\left.\lambda\left(J_{n}\right) \leq n^{-3}\right)$. A straightforward induction establishes the following result.

Lemma 5. Let $h_{1}$ have at most affine growth (i.e. $\left|h_{1}(z)\right| \leq A+B|z|$ ). Then, for each $n \in \mathbb{N}$, all functions in $[H]_{n} \subseteq \mathbb{C}^{[0,1]}$ and thus, in particular, all functions in $[H]_{\infty}=[H]$, belong to $L^{1}(\lambda)$ and attain at most countably many values.

Observe that $[H] \subseteq \mathbb{C}^{[0,1]}$. Consider the linear space $X$ consisting of all functions $f:[0,1] \rightarrow \mathbb{C}$ for which there exists a finite Borel partition $\left\{E_{k}\right\}_{k=1}^{K}$ of $[0,1]$ and functions $g_{k}$ from $Y:=[H]+L^{\infty}(\lambda)$ such that $\left.f\right|_{E_{k}}=\left.g_{k}\right|_{E_{k}}$ for $k=1,2, \ldots, K$.

Lemma 6. We have $L^{\infty}(\lambda) \subseteq X$ and, for each $f \in X$ and each $s \in \mathfrak{B}$, also $s \cdot f \in X$. Moreover, if $h_{1}$ is a Borel function and has at most affine growth, then $X \subseteq L^{1}(\lambda)$ and there exists an $X$-vector measure $\mu$ defined on the Borel subsets of $[0,1]$ for which $X=L^{1}(\mu)$.

Proof. The first claim is routine to establish. The claims about $X$ when $h_{1}$ has at most affine growth follow easily from Lemmas 5 and 3 .

We point out that if $h_{1}$ is continuous and positively homogeneous, then $h_{1}$ is a Borel function and has at most affine growth.

Lemma 7. If $h_{1}$ satisfies a Lipschitz condition, then $h_{1} \circ f \in X$ for each $f \in X$.

Proof. Fix $f \in X$. Choose disjoint Borel sets $E_{1}, \ldots, E_{K}$ (with union $[0,1])$ and corresponding functions $f_{k} \in[H]$ and $b_{k} \in L^{\infty}(\lambda)$ such that $\left.f\right|_{E_{k}}$ is the restriction to $E_{k}$ of $f_{k}+b_{k} \in Y$. Then $\left.\left(h_{1} \circ f\right)\right|_{E_{k}}$ is the restriction to $E_{k}$ of $g_{k}:=h_{1} \circ\left(f_{k}+b_{k}\right)$. Hence, we are done if we can prove that each $g_{k} \in Y$. Since each $h_{1} \circ f_{k} \in[H]$, it suffices to prove that each $g_{k}-\left(h_{1} \circ f_{k}\right)$ is essentially bounded. But, since

$$
\left(g_{k}-\left(h_{1} \circ f_{k}\right)\right)(x)=h_{1}\left(f_{k}(x)+b_{k}(x)\right)-h_{1}\left(f_{k}(x)\right) \quad(x \in[0,1]),
$$

this follows from the fact that each $b_{k}$ is essentially bounded and $h_{1}$ satisfies a Lipschitz condition.

Lemma 8. Let $h_{1}$ and $h_{2}$ be positively homogeneous and continuous. If (13) holds, then $h_{2} \circ H \notin X$. 
Proof. Suppose $h_{2} \circ H \in X$. Then we can write $[0,1]$ as the union of disjoint Borel sets $E_{1}, \ldots, E_{K}$ such that each $\left.\left(h_{2} \circ H\right)\right|_{E_{k}}$ is the restriction to $E_{k}$ of some function from $Y$. By Lemma 2, the latter means that $\left.\left(h_{2} \circ H\right)\right|_{E_{k}}=\left.\left(f_{k} \circ H\right)\right|_{E_{k}}+q_{k}$ a.e. for some $f_{k} \in[I]$ and some bounded measurable function $q_{k}: E_{k} \rightarrow \mathbb{C}$. Since the sets $J_{n}$ are pairwise disjoint, this means that

$$
\sum_{n=1}^{\infty} h_{2}\left(n a_{n}\right) \chi_{J_{n}}=\sum_{n=1}^{\infty} f_{k}\left(n a_{n}\right) \chi_{J_{n}}+q_{k} \quad \text { on } E_{k} \quad(k=1, \ldots, K) .
$$

Since $h_{2}$ and $f_{k}$ are positively homogeneous (see Proposition 6), we obtain

$$
\sum_{n=1}^{\infty} n\left(h_{2}\left(a_{n}\right)-f_{k}\left(a_{n}\right)\right) \chi_{J_{n}}=q_{k} \quad \text { a.e. on } E_{k} \quad(k=1, \ldots, K) .
$$

But, $\left|q_{k}(x)\right| \leq Q$ for some positive constant $Q$ independent of $k$ and $x \in E_{k}$. Since the sets $J_{n}$ are pairwise disjoint, it follows that for any $n, k$ with $\lambda\left(J_{n} \cap E_{k}\right)>0$ the estimate

$$
n\left|h_{2}\left(a_{n}\right)-f_{k}\left(a_{n}\right)\right| \leq Q
$$

holds. For any strictly increasing sequence $n_{1}<n_{2}<\cdots$, we can find some index $k$ and some subsequence $\left(n_{j_{m}}\right)_{m}$ with $\lambda\left(J_{n_{j_{m}}} \cap E_{k}\right)>0$ and thus $\left|h_{2}\left(a_{n_{j_{m}}}\right)-f_{k}\left(a_{n_{j_{m}}}\right)\right| \leq Q / n_{j_{m}} \rightarrow 0$ for $m \rightarrow \infty$. Applying Lemma 4 to the sequences $\left\{b_{k}=\left(f_{k}\left(a_{n}\right)\right)_{n}: k=1, \ldots, K\right\}$, we thus find that $\mathbb{T}$ is the union of closed sets $A_{1}, \ldots, A_{K}$ such that each $\left.h_{2}\right|_{A_{k}}$ is the restriction to $A_{k}$ of a function from $[I]$. Then $\mathbb{C}$ is the union of the closed sets $\hat{A}_{k}:=\bigcup_{\lambda \geq 0}\left(\lambda A_{k}\right)$. Since $h_{2}$ and all functions from $[I]$ are positively homogeneous, it follows that each $\left.h_{2}\right|_{\hat{A}_{k}}$ is the restriction of some function from $[I]$. Hence, (13) fails, which is a contradiction.

Proof of Theorem 2. Let (13) hold. The space $X=L^{1}(\mu)$ defined above (see Lemma 6 ) satisfies (5) by Lemma 6 , satisfies (8) by Lemma 7 , and satisfies (9) by Lemma 8 . Hence, property 2. of Theorem 2 holds. The other implications have been established earlier.

\section{Comparison of conditions (11) and (13)}

As remarked earlier, condition (13) is typically harder to verify than condition (11). One has to prove that $[I]$ does not even contain the function $h_{2}$ "piecewise"; the main difficulty is that one cannot assume anything a priori about the pieces $A_{1}, \ldots, A_{K}$ or their number. We now show, for an important special case, that this condition can be relaxed. This case occurs when the function $h_{1}$ is weakly multiplicative in the following sense: we 
assume for the rest of this section that $h_{1}$ is continuous and that there is a function $m: \mathbb{C} \rightarrow \mathbb{C}$ such that

$$
h_{1}(z w)=m(z) h_{1}(w) \quad(z, w \in \mathbb{C}) .
$$

Unfortunately, condition (14) is rather restrictive, as pointed out by a referee.

Proposition 7. The continuous functions $h_{1}: \mathbb{C} \backslash\{0\} \rightarrow \mathbb{C}$ which satisfy (14) are precisely those of the form

$$
h_{1}(z)=C_{0}|z|^{C_{1}} z^{k}
$$

for fixed $C_{0}, C_{1} \in \mathbb{C}$ and $k \in \mathbb{Z}$.

The only functions of this form which are additionally positively homogeneous are given by

$$
h_{1}(z)=C_{0}|z|\left(\frac{z}{|z|}\right)^{k}
$$

for fixed $C_{0} \in \mathbb{C}$ and $k \in \mathbb{Z}$. Moreover, these functions necessarily satisfy a global Lipschitz condition.

Proof. If we exclude the trivial case that $h_{1}$ is the zero function, then it follows from (14) that $h_{1}(1) \neq 0$ and then that $m(z)=c h_{1}(z)$ for some constant $c \in \mathbb{C} \backslash\{0\}$. In particular, (14) then implies $m(z w)=m(z) m(w)$ and $m(1)=1$. Letting ln denote the principal branch of the logarithm, we can define $g(z):=\ln \left(m\left(e^{z}\right)\right)$ in a circular neighbourhood $U$ of 0 such that $g$ is continuous on $U$ and satisfies on $U$ the Cauchy functional equation $g(z+w)=g(z)+g(w)$. For all $z$ and all $n \in \mathbb{N}$ with $n z \in U$, we thus have $g(n z)=n g(z)$. It follows that $g(w)=n g\left(\frac{1}{n} w\right)$ for all $w \in U$ and all $n \in \mathbb{N}$ and then that $g(q w)=q g(w)$ for all $w \in U$ and rational $q \in[-1,1]$. By continuity, this must hold for all $q \in[-1,1]$. It follows, for all $x \in \mathbb{R}$ in a neighbourhood of 0 , that $g(x)=c_{1} x$ and similarly $g(i x)=c_{2} x$ for constants $c_{1}, c_{2} \in \mathbb{C}$. In particular, for all sufficiently small $x, y \in \mathbb{R}$, we have

$$
\begin{aligned}
m\left(e^{n_{1} x+i n_{2} y}\right) & =m\left(e^{x}\right)^{n_{1}} m\left(e^{i y}\right)^{n_{2}}=e^{n_{1} g(x)} e^{n_{2} g(i y)} \\
& =e^{n_{1} c_{1} x} e^{n_{2} c_{2} y}=e^{c_{1} n_{1} x+c_{2} n_{2} y}
\end{aligned}
$$

for all $n_{1}, n_{2} \in \mathbb{N}$. Since $n_{1}, n_{2} \in \mathbb{N}$ are arbitrary, it follows that actually $m\left(e^{x+i y}\right)=e^{c_{1} x+c_{2} y}$ for all $x, y \in \mathbb{R}$. Since $1=m(1)=m\left(e^{2 \pi i}\right)=e^{2 \pi c_{2}}$, it follows that $c_{2}=i k$ for some $k \in \mathbb{Z}$. Hence, $m\left(e^{x} e^{i y}\right)=m\left(e^{x+i y}\right)=$ $e^{c_{1} x}\left(e^{i y}\right)^{k}$, and so

$$
m(z)=m\left(e^{\ln |z|} \frac{z}{|z|}\right)=e^{c_{1} \ln |z|}\left(\frac{z}{|z|}\right)^{k} .
$$


From this expression (15) is clear with $C_{1}:=c_{1}-k$. If, additionally, $h_{1}$ has the form (15) and is positively homogeneous, then we see (if $C_{0} \neq 0$ ) that $C_{1}+k=1$ from which (16) follows.

To see that the function (16) satisfies a Lipschitz condition, we use the fact that the function $f(z):=z^{k}$ satisfies a Lipschitz condition on the unit circle (because it is continuously real-differentiable). Denoting the corresponding Lipschitz constant by $L$, we have

$$
\begin{aligned}
\left|h_{1}(z)-h_{1}(w)\right| & =\left|C_{0}\right||| z\left|\left(f\left(\frac{z}{|z|}\right)-f\left(\frac{w}{|w|}\right)\right)+(|z|-|w|) f\left(\frac{w}{|w|}\right)\right| \\
& \leq\left|C_{0}\right| L|z|\left|\frac{z}{|z|}-\frac{w}{|w|}\right|+\left|C_{0}\right||z-w| \\
& =\left|C_{0}\right| L \frac{|| w|(z-w)+(|w|-|z|) w|}{|w|}+\left|C_{0}\right||z-w| \\
& \leq\left|C_{0}\right|(2 L+1)|z-w|,
\end{aligned}
$$

and so $h_{1}$ satisfies a global Lipschitz condition.

The reason why we are interested in condition (14) is the following result.

Theorem 3. Assume that $h_{1}, h_{2}: \mathbb{C} \rightarrow \mathbb{C}$ are continuous and that $h_{1}$ satisfies (14). Let $A:=\left\{z \in \mathbb{C}: h_{2}(z) \neq 0\right\}$. Assume, for each function $g: A \rightarrow \mathbb{C}$ which assumes finitely many nonzero values and is constant on the connected components of $A$, that the function $g \cdot\left(\left.h_{2}\right|_{A}\right)$ is not the restriction to $A$ of any function from $[I]$. Then (13) holds for all finite families of Borel sets $A_{1}, \ldots, A_{K}(K \in \mathbb{N})$.

Corollary 3. Assume that $h_{1}, h_{2}: \mathbb{C} \rightarrow \mathbb{C}$ are continuous and that $h_{1}$ satisfies (14). Assume that $A:=\left\{z \in \mathbb{C}: h_{2}(z) \neq 0\right\}$ is dense in $\mathbb{C}$ and, for each function $G: \mathbb{C} \rightarrow \mathbb{C}$ which assumes finitely many nonzero values and is constant on the connected components of $A$, that $G \cdot h_{2} \notin[I]$. Then (13) holds for all Borel sets $A_{1}, \ldots, A_{K}$.

So, in the situation of Corollary 3 , it suffices to verify condition (13) for the sets $A_{1}, A_{2}, \ldots$ which are closures of the components of $A=\{z \in \mathbb{C}$ : $\left.h_{2}(z) \neq 0\right\}$. If there are infinitely many components, then it even suffices to verify condition (13) for all finite families $A_{1}, \ldots, A_{K}$, where $A_{1}, \ldots, A_{K-1}$ are the closures of components of $A$ and $A_{K}:=\overline{\mathbb{C} \backslash\left(A_{1} \cup \cdots \cup A_{K-1}\right)}$. Moreover, in the situation of Corollary 3, to verify (13) it suffices to show that $\left.h_{2}\right|_{A_{k}}$ is not the restriction of the same function from [I] (independent of $k$ ) up to a constant nonzero factor.

Proof. [Proof of Corollary 3] In Theorem 3, the definition of $A$ and the boundedness of $g$ imply that $g \cdot\left(\left.h_{2}\right|_{A}\right)$ has a unique extension to a continuous function on $\bar{A}=\mathbb{C}$. This extension can be written in the form $g \cdot h_{2}$ when 
we put $g=1$ on $\mathbb{C} \backslash A$, for example. Since all functions in $[I]$ are continuous, it suffices to verify that this extension does not belong to $[I]$.

Proof of Theorem 3. Let $a_{n} \in A$ be such that each point of $A$ is the limit of some subsequence of $a=\left(a_{n}\right)_{n}$. Let $S=\mathbb{N}$, equipped with counting measure, and let $\mathcal{F}$ denote the space of all simple functions on $S$, i.e. the set of all complex sequences which attain only finitely many values. Let $X$ denote the linear space of all functions of the form $s \cdot t$ where $s \in \mathcal{F}$ and $t \in[a]$. For any function $G=s \cdot t \in X$, we have by (14) that

$$
h_{1} \circ G=h_{1} \circ(s \cdot t)=(m \circ s) \cdot\left(h_{1} \circ t\right) \in X .
$$

Thus, if we can show that $h_{2} \circ a \notin X$, then the conclusion of Theorem 3 follows from Proposition 5. Assume, by contradiction, that $h_{2} \circ a \in X$. Then $h_{2} \circ a=s \cdot b$, where $b \in[a]$ and $s \in \mathcal{F}$. By Lemma 2 we have $b=f \circ a$ for some $f \in[I]$, i.e. $h_{2} \circ a=s \cdot(f \circ a)$. The sequence $s$ assumes only finitely many distinct values $\lambda_{1}, \ldots, \lambda_{K}$. Applying Lemma 4 to the sequences $b_{k}:=f_{k} \circ a$ with $f_{k}:=\lambda_{k} f$, we find that $A$ is the union of the closed (in $A$ ) sets $B_{k}:=\left\{z \in A: h_{2}(z)=f_{k}(z)\right\}=\left\{z \in A: h_{2}(z)=\lambda_{k} f(z)\right\}$. Note, that in view of $h_{2}(z) \neq 0$ for $z \in B_{k}$, the sets $B_{k}$ are pairwise disjoint. Accordingly, putting $q(z):=\lambda_{k}$ on $B_{k}$, we have $h_{2}=q \cdot f$ and thus $q(z) \neq 0$. Hence, $g:=1 / q$ is defined on $A$ and constant on $B_{k}$ with $g \cdot\left(\left.h_{2}\right|_{A}\right)=\left.f\right|_{A}$ and $f \in[I]$. By construction, the sets where $g$ is constant are closed in $A$. Since these are only finitely many pairwise disjoint sets, they are also open in $A$. Hence, $g$ is constant on the components of $A$. The existence of $g$ contradicts our assumption.

Our previous results can be summarized as follows.

Theorem 4. Let $h_{1}$ be of the form (16) with $C_{0} \in \mathbb{C}$ and $k \in \mathbb{Z}$ (put $\left.h_{1}(0):=0\right)$. Furthermore, assume that $h_{2}: \mathbb{C} \rightarrow \mathbb{C}$ is continuous, positively homogeneous and has at most one zero on the unit circle $\mathbb{T}$. Then the following statements are equivalent.

1. There exists a set $M$ and a complex linear function space $X \subseteq \mathbb{C}^{M}$ satisfying (8) and (9).

2. There exists a vector measure $\mu$ defined on the Borel sets of $[0,1]$ such that $X:=L^{1}(\mu)$ satisfies (5), (8), and (9).

3. Condition (11) holds.

4. Condition (13) holds.

Proof. If (11) holds, then (13) holds by Corollary 3, because $A:=$ $\left\{z: h_{2}(z) \neq 0\right\}$ is dense in $\mathbb{C}$ and consists of only one component. The remaining implications follow from the results of the previous section, if we recall that $h_{1}$ is positively homogeneous, (14) holds and that $h_{1}$ satisfies a global Lipschitz condition (cf. Proposition 7). 
Unfortunately, as we have seen, the three properties used for $h_{1}$ in the proof are already equivalent to (16).

In view of Example 1, we obtain the following consequence.

Corollary 4. There exists a vector measure $\mu$ defined on the Borel sets of $[0,1]$ such that $X:=L^{1}(\mu)$ satisfies (5) and (2) but not (1).

\section{The special case $h_{1}(z)=|z|$}

In order to verify condition (11) for positively homogeneous $h_{1}$, recall that in this case all functions in $[I]$ are positively homogeneous (see Proposition 6). Hence, we may consider $[I]$ as a space of functions on the unit circle $\mathbb{T}$.

Proposition 8. The restrictions of functions from $[I]_{n}$ (resp. $\left.[I]\right)$ to $\mathbb{T}$ are precisely the functions from $\left[\left.I\right|_{\mathbb{T}}\right]_{n}$ (resp. $\left[\left.I\right|_{\mathbb{T}}\right]$ ).

Proof. By Lemma 2 the functions in $\left[\left.I\right|_{\mathbb{T}}\right]_{n}$ (respectively $\left[\left.I\right|_{\mathbb{T}}\right]$ ) are precisely those functions of the form $\left.(f \circ I)\right|_{\mathbb{T}}=\left.f\right|_{\mathbb{T}}$ where $f$ belongs to $[I]_{n}$ (respectively $[I]$ ).

For $h_{1}(z):=|z|$ we are, in view of Theorem 4 , asking the question which positively homogeneous continuous functions $h_{2}: \mathbb{C} \rightarrow \mathbb{C}$ have the property that $\left.h_{2}\right|_{\mathbb{T}} \in\left[\left.I\right|_{\mathbb{T}}\right]$ ? Of course, for this choice of $h_{1}$ there are many functions $h_{2}$ for which it is clear that they do not belong to $[I]$.

Proposition 9. Let $h_{1}(z):=|z|$. Then, considering $h_{1}$ as a function from $\mathbb{R}^{2}$ into $\mathbb{R}^{2}$, all functions in $[I]$ are algebraic, continuous, and positively homogeneous. Moreover, $[I]$ is invariant under inner automorphisms (i.e. for any $c \in \mathbb{C}$ and any $f \in[I]$ the function $z \mapsto f(c z)$ belongs to $[I])$.

Proof. The claims hold even for each $[I]_{n}$ as a straightforward induction on $n$ shows (see also Proposition 6). Concerning the first statement, we actually have more. All functions from $[I]$ are defined by finitely many compositions with the algebraic function $(x, y) \mapsto \sqrt{x^{2}+y^{2}}$ as well as finitely many additions and scalar multiplications.

In particular, for any positively homogeneous, continuous function $h_{2}$ satisfying $h_{2}(z) \neq 0$ for $z \neq 0$ and which fails any one of the above properties, we can construct (by our previous results) a vector measure $\mu$ on the Borel sets of $[0,1]$ such that $L^{1}(\mu)$ is a complex lattice (and contains $\left.L^{\infty}(\mu)\right)$ but for which there exists some $f \in L^{1}(\mu)$ with $h_{2} \circ f \notin L^{1}(\mu)$.

In view of the open problem mentioned in the introduction, the function $h_{2}(z)=\bar{z}$ (or equivalently $h_{2}(z)=\operatorname{Re} z$ ) would be of a particular interest in this context. Unfortunately, this function satisfies all of the properties of 
Proposition 9, and we know of no way of answering the question of whether $\left.h_{2}\right|_{\mathbb{T}} \in\left[\left.I\right|_{\mathbb{T}}\right]$ ?

It is hard to decide for particular functions, satisfying the properties of Proposition 9 , whether or not they are contained in $\left[\left.I\right|_{\mathbb{T}}\right]$. Indeed, the space $\left[\left.I\right|_{\mathbb{T}}\right]$ is dense in the space $C(\mathbb{T})$ of all continuous complex functions on $\mathbb{T}$ (with the max-norm) as the following result shows. So, to decide whether a function belongs to $\left[\left.I\right|_{\mathbb{T}}\right]$ is a rather "fine" property of the function.

Theorem 5. Let $h_{1}(z):=|z|$. Then the closure of $\left[\left.I\right|_{\mathbb{T}}\right]_{3}$ in $C(\mathbb{T})$ contains $\left.\operatorname{Re}(\cdot)\right|_{\mathbb{T}}$ (and thus also $\left.z \mapsto \bar{z}\right)$. Moreover, $\left[\left.I\right|_{\mathbb{T}}\right]$ is dense in $C(\mathbb{T})$.

Proof. Consider the sequence $f_{n}(z):=|z+n|-|z-n|$, which belongs to $\left[\left.I\right|_{\mathbb{T}}\right]_{3}$. Then

$$
(|z+n|+|z-n|) f_{n}(z)=4 n \operatorname{Re} z,
$$

and so a straightforward estimate shows that the sequence $g_{n}(z):=f_{n}(z)-z$ from $\left[\left.I\right|_{\mathbb{T}}\right]_{3}$ converges uniformly on $\mathbb{T}$ to $z \mapsto(2 \operatorname{Re} z)-z$.

For the second claim, let $X$ denote the real subspace of all $\mathbb{R}$-valued functions in $\left[\left.I\right|_{\mathbb{T}}\right]$. Then $X$ contains the real constant functions, and the two functions $g(z):=|z+1|$ and $h(z):=|z+i|$ from $X$ separate points of $\mathbb{T}$. Indeed, if $u=u_{1}+i u_{2}$ and $w=w_{1}+i w_{2}$ are distinct points of $\mathbb{T}$, then $\varphi:=\left(u_{1}-w_{1}\right) g+\left(u_{2}-w_{2}\right) h$ satisfies $\varphi(u) \neq \varphi(w)$. Moreover, $X$ has property (1), and so the maximum and minimum of each finite collection of functions from $X$ is also in $X$. Since $\mathbb{T}$ is compact, an argument completely analogous to the standard proof of the Stone-Weierstrass theorem (e.g. the Lemma in [3, Section 36]) shows that $X$ is dense in the space of all $\mathbb{R}$-valued continuous functions on $\mathbb{T}$. In particular, $X+i X \subseteq\left[\left.I\right|_{\mathbb{T}}\right]$ is dense in the space of all complex continuous functions on $\mathbb{T}$.

In view of Propositions 3 and 6 , the functions $h_{2}$ which belong to $[I]$ (still for $\left.h_{1}(z):=|z|\right)$ can also be characterized geometrically if we interpret $\mathbb{C}$ as the plane. These are precisely those positively homogeneous functions $h_{2}$ with the property that there is a universal construction which gives for any $z \in \mathbb{T}$ the value $h_{2}(z) \in \mathbb{C}$ using only a finite number of the following operations:

1. Stretching a point (from 0) by a fixed factor. (Here, "fixed" means independent of $z$ ).

2. Rotating a point (around 0 ) by a fixed angle.

3. Rotating a point (around 0) to the positive real axis.

4. Addition of two points obtained from a previous step.

Thus, the (still open) question mentioned in the introduction is equivalent to the question of whether the conjugate function $h_{2}(z)=\bar{z}$ or, equivalently, $h_{2}(z)=\operatorname{Re} z$, can be constructed in this way. 
Acknowledgements. The authors wish to thank Dr. S. Okada for some useful and relevant discussions.

\section{References}

[1] S. Okada and W. J. Ricker, Vector measures and integration in noncomplete spaces, Arch. Math. (Basel), 63 (1994), 344-353.

[2] W. Rudin, Real and complex analysis, 3rd ed., McGraw-Hill, Singapore, 1987.

[3] G. F. Simmons, Introduction to topology and modern analysis, McGraw -Hill, Tokyo, 1963.

Math.-Geogr. Fakultät

Katholische Universität Eichstätt-Ingolstadt

D-85071 Eichstätt

Germany

(Email : werner.ricker@ku-eichstaett.de)

University of Würzburg

Department of Mathematics

Am Hubland

D-97074 Würzburg

Germany

(Email : vaeth@mathematik.uni-wuerzburg.de)

(Received : December 2002) 


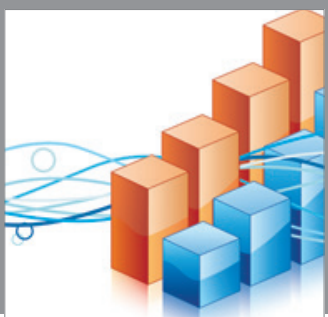

Advances in

Operations Research

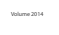

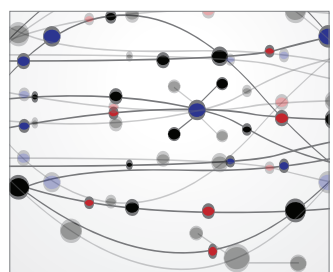

\section{The Scientific} World Journal
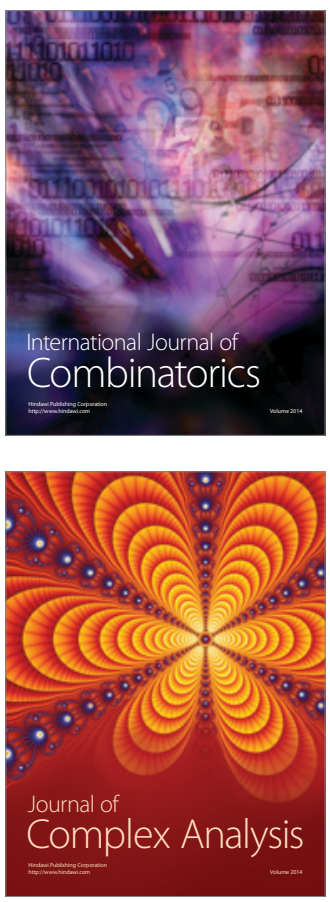

International Journal of

Mathematics and

Mathematical

Sciences
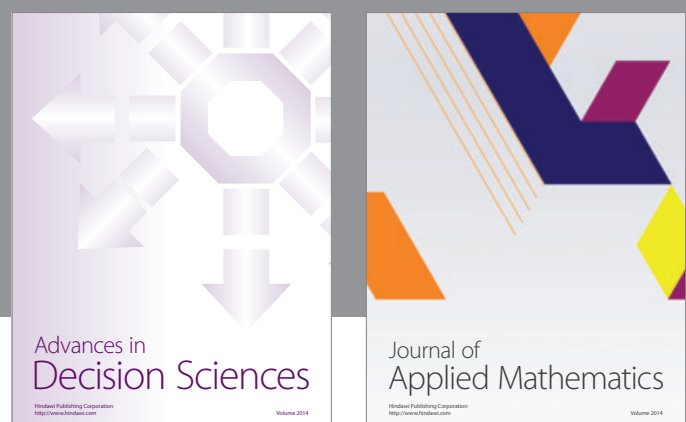

Journal of

Applied Mathematics
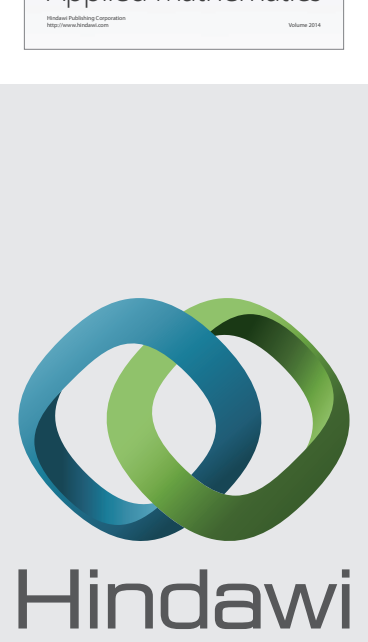

Submit your manuscripts at http://www.hindawi.com
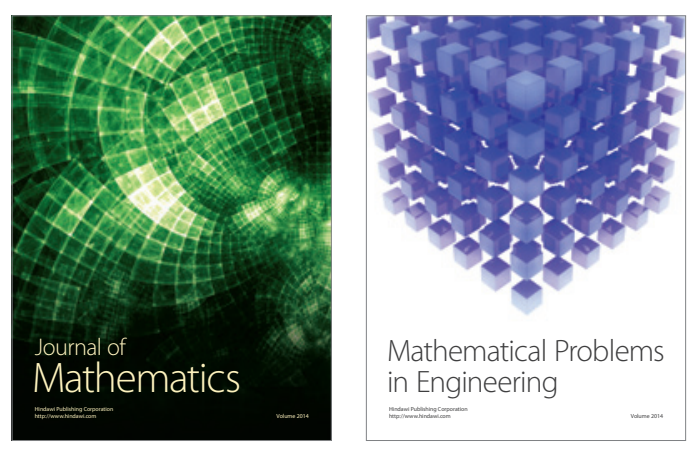

Mathematical Problems in Engineering
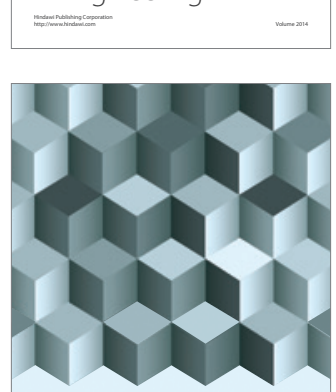

Journal of

Function Spaces
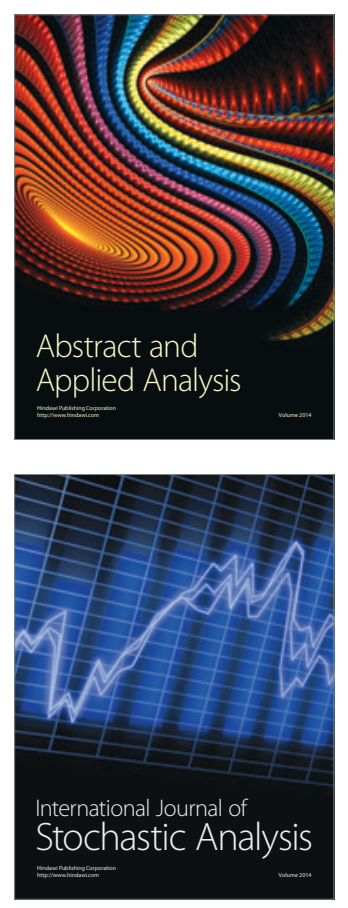

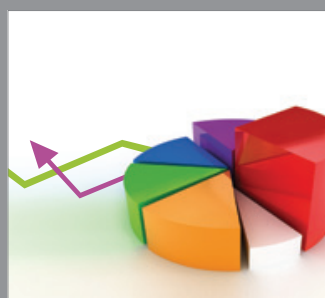

ournal of

Probability and Statistics

Promensencen
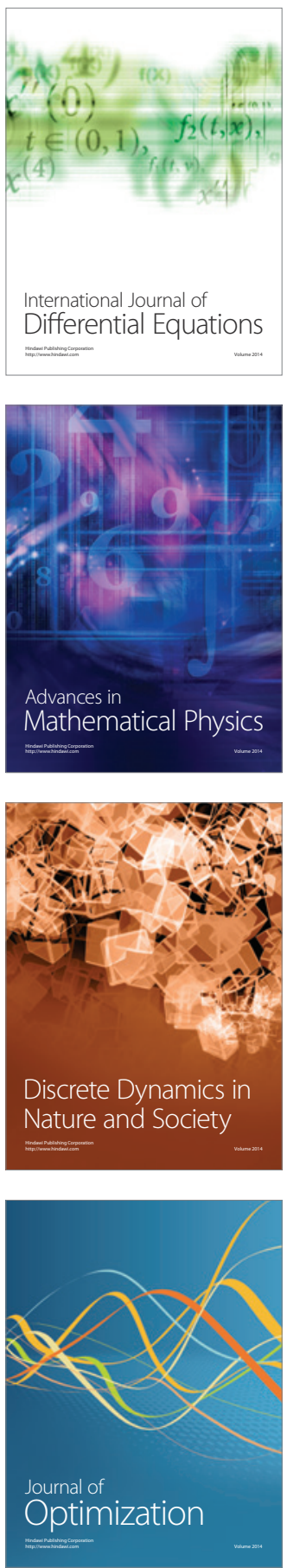